M. Najariyan, M. H. Farahi, and M. Alavian / TJMCS Vol .2 No.4 (2011) 639-649

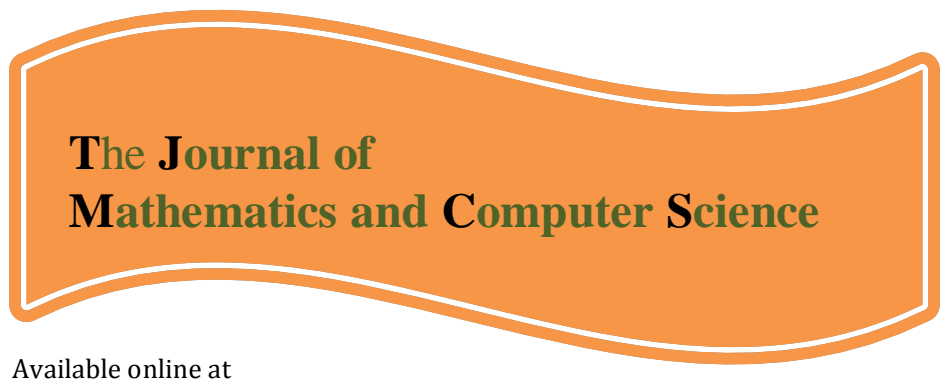

http://www.TJMCS.com

The Journal of Mathematics and Computer Science Vol .2 No.4 (2011) 639-649

\title{
Optimal Control of HIV Infection by using Fuzzy Dynamical Systems
}

\author{
M. Najariyan1, ${ }^{*}$, M. H. Farahi², and M. Alavian \\ School of Mathematics, Ferdowsi University of Mashhad, Iran, ma_na_707@yahoo.com \\ School of Mathematics, Ferdowsi University of Mashhad, Iran, and the center of excellence in modelling and computations in \\ linear and nonlinear systems (CRMCS), Farahi@math.um.ac.ir \\ Islamic Azad University, Mashhad, Iran, mojgan.alavian@gmail.com
}

\begin{abstract}
A dynamical system represent the infection and propagation of HIV is considered. First a mathematical model for the HIV is simulated. Since age, sex... are important parameters in treatment of HIV disease, it is natural to consider the variables as fuzzy variables. Thus we need to consider a fuzzy dynamical system to control the HIV disease. To solve such a fuzzy dynamical system, by using $\alpha$-cuts, one can convert this system to a non-fuzzy system of differential equations, then by using numerical methods one may attempts to solve these differential equations
\end{abstract}

Keywords: Fuzzy differential equations, nonlinear programming, discretization method, HIV

\section{Introduction}

A basic model of virus dynamic was introduced by Nowak and May [1]. This model has served as a framework to build other models of HIV infection, also according to the Painleve' property (PP) test [2], the Nowak-May model is completely integrable. In [3] is proposed a class of models based on the NowakMay model to study reactivation of resting infected T-cells, $T_{r}$, during long-term HIV infection. These models incorporate classes of $T_{r}$ cells and cytotoxic T-cells, $T_{c}$. A number of mathematical models have been proposed to understand HIV dynamics, disease process, anti-retroviral response etc. [4]-[7]. In [8] the existence and local stability of the infected steady state are studied. Also the global stability of infected steady state is discussed. The dynamic multidrug therapy problem is modeled in [9] as an optimal control model that maximizes the inhibition of HIV. In [10], finite horizon open loop control tools are applied to an HIV chemotherapy model using an objective function based on a combination of maximizing T-cells

\footnotetext{
1,* PhD student control and optimal control

${ }^{2}$ Professor in optimization and control theory
} 
count and minimizing the systemic cost of chemotherapy. In [11] the authors introduced a control and drug dosage for the HIV infection. Several authors have advocated the use of fuzzy set theory in epidemiology problems [12]-[15] and population dynamics [16].

In this paper we study the Nowak-May model with fuzzy variables and introduce the fuzzy control model to maximizing uninfected cells of HIV disease.

\section{Preliminaries}

In this section we give some definitions and introduce the necessary notations used later. For details see [17]-[21].

Definition 1. Let $X$ be a nonempty set in $\mathfrak{R}$, a fuzzy set $\mathrm{u}$ in $X$ is a mapping $u: \mathfrak{R} \rightarrow[0,1]$, we denote by $E^{1}$ the class of fuzzy subsets satisfying the following properties:

- $u$ is normal, i.e. there exists $x \in \mathfrak{R}$, such that $u(x)=1$.

- $u$ is fuzzy convex, i.e. $\forall x, y \in \mathfrak{R}$ and $0 \leq \lambda \leq 1, u(\lambda x+(1-\lambda) y) \geq \min \{u(x), u(y)\}$.

- $u$ is upper semi continuous.

- $\quad c l[u]^{0}=\{s \in \mathfrak{R} \mid u(s)>0\}$, is compact, where $c l$ denotes the closure of subsets.

For $0<\alpha \leq 1$, denote $[u]^{\alpha}=\{s \mid u(s) \geq \alpha\},[u]^{\alpha}$ is $\alpha$-level set. If $u \in E^{1}$, then $[u]^{\alpha}=\left[\underline{u}^{\alpha}, \bar{u}^{\alpha}\right]$, where $\underline{u}^{\alpha}=\inf \{x \in \mathfrak{R}: u(x) \geq \alpha\}>-\infty$ and $\bar{u}^{\alpha}=\sup \{x \in \mathfrak{R}: u(x) \geq \alpha\}<\infty$.

For $u, v \in E^{1}$ and $\gamma \in \mathbb{R}$ the sum $u \oplus v$ and the product $\gamma \odot u$ defined by $[u \oplus v]^{\alpha}=[u]^{\alpha}+[v]^{\alpha}$ and $[\gamma \odot u]^{\alpha}=\gamma \cdot[u]^{\alpha}$ for all $\alpha \in[0,1]$.

The metric space is given by the Housdorf distance

$$
\begin{aligned}
& D: E^{1} \times E^{1} \rightarrow \mathfrak{R}^{+} \cup\{0\} \\
& D(u, v)=\sup _{\alpha \in[0,1]} \max \left\{\left|\underline{u}^{\alpha}-\underline{v}^{\alpha}\right|,\left|\bar{u}^{-\alpha}-\bar{v}^{\alpha}\right|\right\}
\end{aligned}
$$

$\left(E^{1}, D\right)$ is a complete metric space.

Definition 2. Let $x, y \in E^{1}$ if there exists $z \in E^{1}$ such that $x=y \oplus z$ then $z$ is called the H-difference of $x, y$ and it is denoted by $x \ominus y$.

Definition 3. Let $\tilde{f}: T \subseteq \mathfrak{R} \rightarrow E^{1}$ and $x_{0} \in T$ is fixed, then:

- $f$ is (1)-differentiable at $x_{0} \in T$ if for $h>0$ sufficiently small there exist the H-differences

$f\left(x_{0}+h\right) \ominus f\left(x_{0}\right)$ and $f\left(x_{0}\right) \ominus f\left(x_{0}-h\right)$ and the limits (in the metric D):

$$
\lim _{h \rightarrow 0^{+}} \frac{f\left(x_{0}+h\right) \ominus f\left(x_{0}\right)}{h}=\lim _{h \rightarrow 0^{+}} \frac{f\left(x_{0}\right) \ominus f\left(x_{0}-h\right)}{h}=f^{x}\left(x_{0}\right)
$$

- $f$ is (2)-differentiable at $x_{0} \in T$ if for all $h>0$ sufficiently small there exist the H-differences

$$
f\left(x_{0}-h\right) \ominus f\left(x_{0}\right) \text { and } f\left(x_{0}\right) \ominus f\left(x_{0}+h\right) \text { and the limits (in the metric D): }
$$

$$
\lim _{h \rightarrow 0^{+}} \frac{f\left(x_{0}\right) \ominus f\left(x_{0}+h\right)}{(-h)}=\lim _{h \rightarrow 0^{+}} \frac{f\left(x_{0}-h\right) \ominus f\left(x_{0}\right)}{(-h)}=f^{\prime}\left(x_{0}\right)
$$

If $f$ is (n)-differentiable at $x_{0}$, we denote its first derivatives by $D_{n}^{(1)} f\left(x_{0}\right)$ for $n=1,2$.

Example 1. Let $g: T \rightarrow \mathbb{R}^{+}$and define $f: T \rightarrow E^{1}$ by $f(x)=c \odot g(x)$ where $x \in T$ and $c$ is a fuzzy number. If $g$ is differentiable at $x_{0} \in T$, then $f$ is (1)-differentiable if $g^{\prime}\left(x_{0}\right)>0$ and $f$ is (2)differentiable at $x_{0}$ if $g^{\prime}\left(x_{0}\right)<0$. 
Theorem 1. Let $f: T \rightarrow E^{1}$ be a fuzzy function, where $[f(x)]^{\alpha}=\left[f^{\alpha}(x), \bar{f}^{\alpha}(x)\right]$,

- If $f$ is (1)-differentiable, then $f^{\alpha}(x)$ and $\bar{f}^{-\alpha}(x)$ are differentiable functions and

$$
\left[D_{1}^{(1)} f(x)\right]^{\alpha}=\left[\underline{f}^{\prime \alpha}(x), \bar{f}^{\prime \alpha}(x)\right]
$$

- If $f$ is (2)-differentiable, then $\underline{f}^{\alpha}(x)$ and $\bar{f}^{-\alpha}(x)$ are differentiable functions and

$$
\left[D_{2}^{(1)} f(x)\right]^{\alpha}=\left[\bar{f}^{\prime \alpha}(x), \underline{f}^{\prime \alpha}(x)\right]
$$

Proof: See [20].

Let us consider the following fuzzy initial value differential equations with initial value condition

$$
\begin{aligned}
& \dot{\tilde{x}}(t)=\tilde{f}(\tilde{x}(t), t) . \quad t_{0} \leq t \leq t_{f} \\
& \tilde{x}\left(t_{0}\right)=\tilde{x}_{0}
\end{aligned}
$$

where $\tilde{f}: E^{1} \times I \rightarrow E^{1}$ is a continuous fuzzy mapping and $\tilde{x}_{0}$ is a fuzzy number.

Theorem 2. Let $\tilde{f}: E^{1} \times I \rightarrow E^{1}$ be a continuous fuzzy function such that there exists $\mathrm{k}>0$ such that $D(\tilde{f}(t, \tilde{x}), \tilde{f}(t, \tilde{z})) \leq k D(\tilde{x}, \tilde{z})$ for all $t \in I$ and $\tilde{x}, \tilde{z} \in E^{1}$, Then problem (1) has two solutions (one (1)differentiable and the other one (2)-differentiable) on I, where $I=\left[t_{0}, t_{f}\right]$.

We assume $I=\left[t_{0}, t_{f}\right]$.

Proof. See [22].

By using Theorems 1-2 the problem (1) has the following forms:

- If $f$ is (1)-differentiable then

$$
\begin{cases}\dot{\bar{x}}^{\alpha}(t)=\bar{f}^{\alpha}\left(t, \bar{x}^{\alpha}, \underline{x}^{\alpha}\right), & \bar{x}^{\alpha}\left(t_{0}\right)=\bar{x}_{0}{ }^{\alpha} \\ \underline{\dot{x}}^{\alpha}(t)=\underline{f}^{\alpha}\left(t, \bar{x}^{\alpha}, \underline{x}^{\alpha}\right), & \underline{x}^{\alpha}\left(t_{0}\right)=\underline{x}_{0}{ }^{\alpha}\end{cases}
$$

- If $f$ is (2)-differentiable then

$$
\begin{cases}\dot{\bar{x}}^{\alpha}(t)=f^{\alpha}\left(t, \bar{x}^{\alpha}, \underline{x}^{\alpha}\right), & \bar{x}^{\alpha}\left(t_{0}\right)=\bar{x}_{0}{ }^{\alpha} \\ \underline{\dot{x}}^{\alpha}(t)=\overline{\bar{f}}^{\alpha}\left(t, \bar{x}^{\alpha}, \underline{x}^{\alpha}\right), & \underline{x}^{\alpha}\left(t_{0}\right)=\underline{x}_{0}{ }^{\alpha} .\end{cases}
$$

The generalized Euler method based on the first-order approximation of $\dot{\bar{x}}^{\alpha}(t)$ and $\underline{\dot{x}}^{\alpha}(t)$, denoted in [23] and represented as follow:

$$
\begin{aligned}
& \begin{cases}\bar{x}^{\alpha}\left(t_{i+1}\right)=\bar{x}^{\alpha}\left(t_{i}\right)+h \times \bar{f}^{\alpha}\left(t, \bar{x}^{\alpha}, \underline{x}^{\alpha}\right) & \bar{x}^{\alpha}\left(t_{0}\right)=\bar{x}_{0}{ }^{\alpha} \\
\underline{x}^{\alpha}\left(t_{i+1}\right)=\underline{x}^{\alpha}\left(t_{i}\right)+h \times \underline{f}^{\alpha}\left(t, \bar{x}^{\alpha}, \underline{x}^{\alpha}\right) & \underline{x}^{\alpha}\left(t_{0}\right)=\underline{x}_{0}{ }^{\alpha}\end{cases} \\
& \begin{cases}\bar{x}^{\alpha}\left(t_{i+1}\right)=\bar{x}^{\alpha}\left(t_{i}\right)+h \times \bar{f}^{\alpha}\left(t, \bar{x}^{\alpha}, \underline{x}^{\alpha}\right) & \bar{x}^{\alpha}\left(t_{0}\right)=\bar{x}_{0}{ }^{\alpha} \\
\underline{x}^{\alpha}\left(t_{i+1}\right)=\underline{x}^{\alpha}\left(t_{i}\right)+h \times \bar{f}^{\alpha}\left(t, \bar{x}^{\alpha}, \underline{x}^{\alpha}\right) & \underline{x}^{\alpha}\left(t_{0}\right)=\underline{x}_{0}{ }^{\alpha}\end{cases}
\end{aligned}
$$

where $h=\frac{t_{f}-t_{0}}{N}$ and $t_{i}=t_{0}+i \times h$ for $i=1, \ldots, N$.

\section{Disceretization method}

Consider the following controlled system: 


$$
\begin{aligned}
& \dot{x}(t)=f(x(t), u(t), t) . \quad t_{0} \leq t \leq t_{f} \\
& x\left(t_{0}\right)=x_{0}
\end{aligned}
$$

where the trajectory function $t \in\left[t_{0}, t_{f}\right] \rightarrow x(t) \in A_{1} \subseteq R^{n}$ is absolutely continuous and the control function $t \in\left[t_{0}, t_{f}\right] \rightarrow u(t) \in U_{1} \subseteq R^{m}$, is Lebesgue- measurable where $A_{1}$ and $U_{1}$ are compact sets. The equation (2) is the differential equation describing the controlled system. In sequel, without losing generality, we assume $t_{0}=0$, and $t_{f}=1$.

Definition 4. Global error function in controlled system (2) is defined in $\mathfrak{R}^{n+m+1}$ space as below: $E(x(t), u(t), t)=\int_{0}^{1}\|\dot{x}(t)-f(x(t), u(t), t)\| d t$,

where $f: A_{1} \times U_{1} \times[0,1] \rightarrow R^{n}$ is a linear or nonlinear continuous function of $t, x$ and $u$, and $\|$. $\|$ is a norm function defined in $\mathfrak{R}^{n+m+1}$ space.

Theorem 3. The ordered pair $(x(t), u(t))$ is a solution of the controlled system (2) with boundary condition $x(0)=x_{0}$ if and only if $E(x(t), u(t), t)=0$.

Proof. See [24].

Via Theorem 3 one can consider the following optimization problem:

$$
\operatorname{Min} \int_{0}^{1}\|\dot{x}(t)-f(x(t), u(t), t)\| d t
$$

s.t.

$$
x\left(t_{0}\right)=x_{0}
$$

To solve (3) by discretization, divide the interval [0,1] into $N$ equidistant parts and assume $k=\frac{1-0}{N}$, the discretization form of the problem (3) is as follows:

$$
\operatorname{Min} \sum_{i=0}^{N-1}\left\|\dot{x}\left(t_{i}\right)-f\left(x\left(t_{i}\right), u\left(t_{i}\right), t_{i}\right)\right\| \times k
$$

s.t.

$$
x\left(t_{0}\right)=x_{0}
$$

where $\left\|\dot{x}\left(t_{i}\right)-f\left(x\left(t_{i}\right), u\left(t_{i}\right), t_{i}\right)\right\|=\sum_{j=1}^{n}\left|\dot{x}_{j}\left(t_{i}\right)-f_{j}\left(x\left(t_{i}\right), u\left(t_{i}\right), t_{i}\right)\right|$. Now by using the forward difference to approximate $\dot{x}\left(t_{i}\right)$, i.e.

$$
\dot{x}\left(t_{i}\right)=\frac{x\left(t_{i+1}\right)-x\left(t_{i}\right)}{k},
$$

and replacing (5) in (4) we have:

$$
\operatorname{Min} \sum_{i=0}^{N-1}\left\|\frac{x\left(t_{i+1}\right)-x\left(t_{i}\right)}{k}-f\left(x\left(t_{i}\right), u\left(t_{i}\right), t_{i}\right)\right\| \times k
$$

s.t. 


$$
x\left(t_{0}\right)=x_{0} .
$$

We need to mention that if $x$ is a free variable then, one can write $x=y-z$ and $|x|=y+z$ where y and $z$ are nonnegative variables.

Via this mention we define:

$$
\frac{x_{j}\left(t_{i+1}\right)-x_{j}\left(t_{i}\right)}{k}-f_{j}\left(x\left(t_{i}\right), u\left(t_{i}\right), t_{i}\right)=y_{i j}-z_{i j},
$$

so

$$
\left|\frac{x_{j}\left(t_{i+1}\right)-x_{j}\left(t_{i}\right)}{k}-f_{j}\left(x\left(t_{i}\right), u\left(t_{i}\right), t_{i}\right)\right|=y_{i j}+z_{i j} \text {. }
$$

By replacing the above absolute value in (6), we find the following optimization problem:

$$
\operatorname{Min} \sum_{i=0}^{N-1} \sum_{j=1}^{n}\left(y_{i}+z_{i}\right) \times k
$$

s.t.

$$
\begin{aligned}
& \frac{x_{j}\left(t_{i+1}\right)-x_{j}\left(t_{i}\right)}{k}-f_{j}\left(x\left(t_{i}\right), u\left(t_{i}\right), t_{i}\right)=y_{i j}-z_{i j}, \quad i=0, \ldots, N-1, j=1, \ldots, n \\
& x(0)=x_{0}
\end{aligned}
$$

In the next section, we use the above scheme to control the fuzzy model of HIV infection.

\section{Fuzzy model for HIV infection}

Consider the following equations for HIV infection (Nowak-May Model [1])

$$
\begin{aligned}
& \frac{d n}{d t}=r-a n-\beta n v \\
& \frac{d i}{d t}=\beta n v-b i \\
& \frac{d v}{d t}=k i-s v,
\end{aligned}
$$

The parameters and initial values are shown respectively in Table 1 and 2.

Table 1. Parameters of the microscopic HIV model

\begin{tabular}{|c|c|c|}
\hline$r=7$ & $a=0.007$ & $\beta=42163 \times 10^{-11}$ \\
\hline$b=0.0999$ & $s=0.2$ & $k=90.67$ \\
\hline
\end{tabular}

Table 2. Initial conditions of the crisp model

\begin{tabular}{|c|c|}
\hline$n(0)$ & 1000 \\
\hline$i(0)$ & 5 \\
\hline$v(0)$ & 7000 \\
\hline tinitial & 0 time units \\
\hline t final & 500 time units \\
\hline
\end{tabular}

where $n$ denotes the uninfected cell, $i$ denotes infected cells and $v$ is the number free virus particles. Uninfected cells are produced at a constant rate, $r$, and die at a rate, an, and uninfected cells with free virus produce the infected cells at rate, $\beta n v$, in the second equation infected cells die at rate, $b i$, and in the thired equation free virus is produced from infected cells at rate, $k i$, and die at rate, $s v$. 
Since age, sex, feeding,... are important parameters in treatment of HIV disease, it is natural to consider the variables as fuzzy variables, the fuzzy form of differential equation system (7) is as bellow:

$$
\begin{aligned}
& \frac{d \tilde{n}}{d t}=r-a \tilde{n}-\beta \tilde{n} \tilde{v} \\
& \frac{d \tilde{i}}{d t}=\beta \tilde{n} \tilde{v}-b \tilde{i} \\
& \frac{d \tilde{v}}{d t}=k \tilde{i}-s \tilde{v}
\end{aligned}
$$

where the initial conditions of the fuzzy model are shown in Table 3. Table 3. Initial conditions of the fuzzy model

\begin{tabular}{|c|c|}
\hline$\tilde{n}(0)$ & $(850,1000,1150)$ \\
\hline$\tilde{i}(0)$ & $(3,5,7)$ \\
\hline$\tilde{v}(0)$ & $(6750,7000,7250)$ \\
\hline
\end{tabular}

Now by using Theorem 1, the system of fuzzy differential equations (8) changes to the two crisp differential equations systems as follows:

$$
\begin{aligned}
& \frac{d \bar{n}^{\alpha}}{d t}=r-a \underline{n}^{\alpha}-\beta \underline{n}^{\alpha} \underline{v}^{\alpha} \\
& \frac{d \bar{i}^{\alpha}}{d t}=\beta \bar{n}^{-\alpha-\alpha} v-b \underline{i}^{\alpha} \\
& \frac{d \bar{v}}{d t}=k \bar{i}^{\alpha}-s \underline{v}^{\alpha}
\end{aligned}
$$

and

$$
\begin{aligned}
& \frac{d \underline{n}^{\alpha}}{d t}=r-a \bar{n}^{\alpha}-\beta \bar{n}^{\alpha-\alpha} v \\
& \frac{d \underline{i}^{\alpha}}{d t}=\beta \underline{n}^{\alpha} \underline{v}^{\alpha}-b i^{-\alpha} \\
& \frac{d \underline{v}^{\alpha}}{d t}=k \underline{i}^{\alpha}-s \bar{v}
\end{aligned}
$$

or

$$
\begin{aligned}
& \frac{d \bar{n}^{\alpha}}{d t}=r-a \bar{n}^{\alpha}-\beta \bar{n}^{-\alpha-\alpha} \bar{v}^{-\alpha} \\
& \frac{d \bar{i}^{\alpha}}{d t}=\beta \underline{n}^{\alpha} \underline{v}^{\alpha}-b \bar{i}^{\alpha} \\
& \frac{d \bar{v}^{\alpha}}{d t}=k \underline{i}^{\alpha}-s \bar{v}
\end{aligned}
$$

and 


$$
\begin{aligned}
& \frac{d \underline{n}^{\alpha}}{d t}=r-a \underline{n}^{\alpha}-\beta \underline{n}^{\alpha} \underline{v}^{\alpha} \\
& \frac{d \underline{i}^{\alpha}}{d t}=\beta \bar{n}{ }^{\alpha-\alpha} v-b \underline{i}^{\alpha} \\
& \frac{d \underline{v}^{\alpha}}{d t}=k \bar{i}^{\alpha}-s \underline{v}^{\alpha}
\end{aligned}
$$

Table 4. $\alpha$-cut of the initial conditions

\begin{tabular}{|c|c|}
\hline $\bar{n}^{\alpha}(0)$ & $1000 \alpha+1150(1-\alpha)$ \\
\hline$\underline{n}^{\alpha}(0)$ & $1000 \alpha+850(1-\alpha)$ \\
\hline $\bar{i}^{\alpha}(0)$ & $5 \alpha+6(1-\alpha)$ \\
\hline$\underline{i}^{\alpha}(0)$ & $5 \alpha+4(1-\alpha)$ \\
\hline $\bar{v}^{\alpha}(0)$ & $7000 \alpha+7250(1-\alpha)$ \\
\hline$\underline{v}^{\alpha}(0)$ & $7000 \alpha+6750(1-\alpha)$ \\
\hline
\end{tabular}

Using generalized Euler method for solving two systems (9.a) and (9.b) will show the systems are not converges. While the solution of the two systems (10.a) and (10.b) have the following convergence figure:
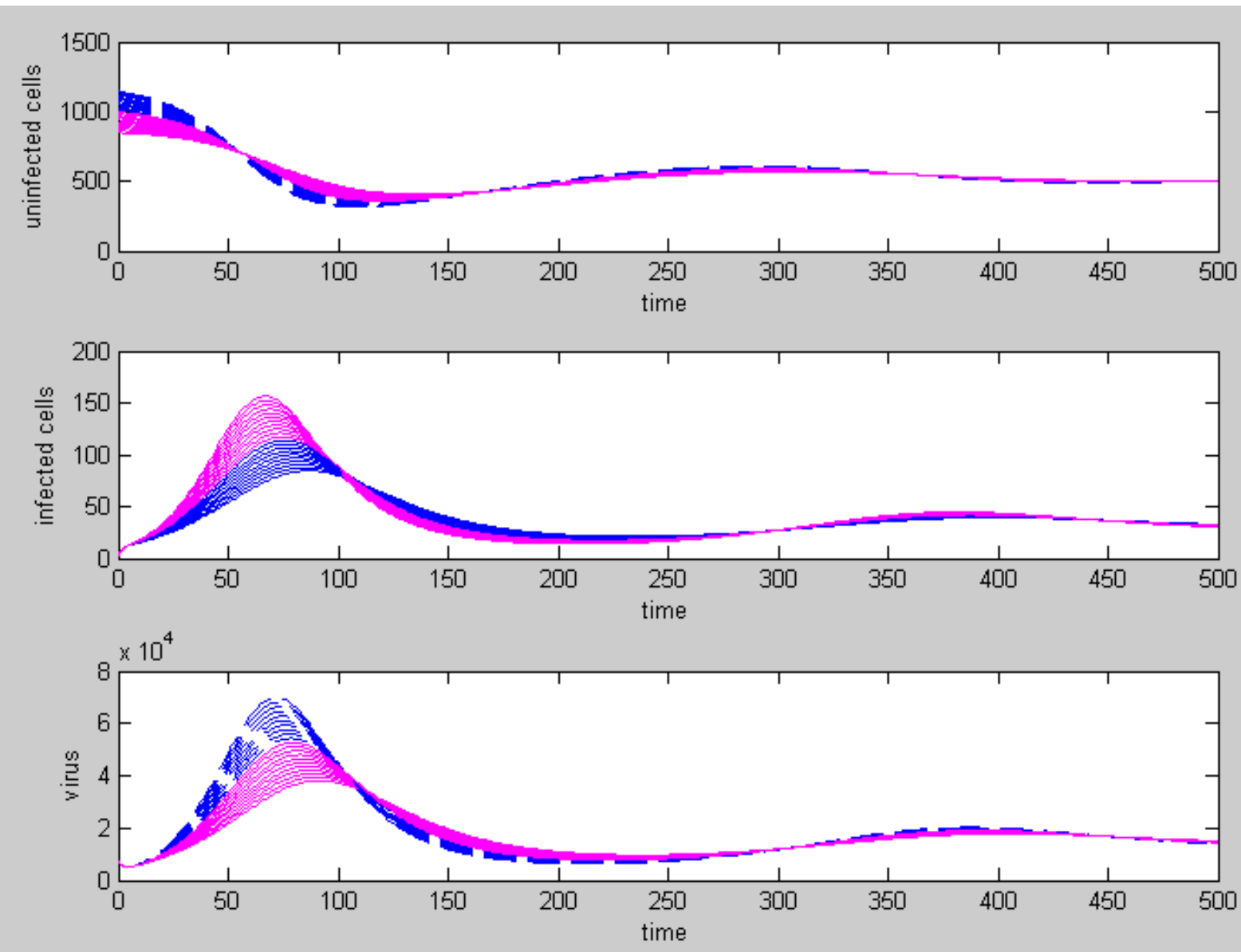

Figure 1. The solution for systems (10.a) and (10.b) 
Now we define the control function $u(t)$ to prevent the uninfected cells, thus we consider the following control system:

$$
\begin{aligned}
& \frac{d n}{d t}=r-a n-\beta n v(1-u) \\
& \frac{d i}{d t}=\beta n v(1-u)-b i \\
& \frac{d v}{d t}=k i-s v
\end{aligned}
$$

where $0 \leq u \leq 1$.

The fuzzy form of the dynamical control system (11) is as follows:

$$
\begin{aligned}
& \frac{d \tilde{n}}{d t}=r-a \tilde{n}-\beta \tilde{n} \tilde{v}(1-u) \\
& \frac{d \tilde{i}}{d t}=\beta \tilde{n} \tilde{v}(1-u)-b \tilde{i} \\
& \frac{d \tilde{v}}{d t}=k \tilde{i}-s \tilde{v}
\end{aligned}
$$

By using the Theorem1 the above system change to the non-fuzzy system of differential equations as:

$$
\begin{aligned}
& \frac{d \bar{n}^{\alpha}}{d t}=r-a \bar{n}^{-\alpha}-\beta \bar{n} \bar{v}^{-\alpha}(1-u) \\
& \frac{d \bar{i}^{\alpha}}{d t}=\beta \underline{n}^{\alpha} \underline{v}^{\alpha}(1-u)-b i^{-\alpha} \\
& \frac{d \bar{v}^{\alpha}}{d t}=k \underline{i}^{\alpha}-s{ }^{-\alpha}
\end{aligned}
$$

and

$$
\begin{aligned}
& \frac{d \underline{n}^{\alpha}}{d t}=r-a \underline{n}^{\alpha}-\beta \underline{n}^{\alpha} \underline{v}^{\alpha}(1-u) \\
& \frac{d \underline{i}^{\alpha}}{d t}=\beta \bar{n}^{\alpha-\alpha} v(1-u)-b \underline{i}^{\alpha} \\
& \frac{d \underline{v}^{\alpha}}{d t}=k i^{\alpha}-s \underline{v}^{\alpha}
\end{aligned}
$$

To solve (13.a)-(13.b), we used the discretization method described in Section 3. Defined $h=\frac{500-0}{n}$, so we reach the following nonlinear optimization problem: 
$\operatorname{Min} \sum_{j=0}^{n-1}\left(y_{j}^{1}+z_{j}^{1}+y_{j}^{2}+z_{j}^{2}+y_{j}^{3}+z_{j}^{3}+y_{j}^{4}+z_{j}^{4}+y_{j}^{5}+z_{j}^{5}+y_{j}^{6}+z_{j}^{6}\right) \times h$

s.t.

$\frac{\bar{n}^{\alpha}\left(t_{j+1}\right)-\bar{n}^{\alpha}\left(t_{j}\right)}{h}-\left(r-a \bar{n}^{\alpha}\left(t_{j}\right)-\beta \bar{n}^{\alpha}\left(t_{j}\right) \bar{v}^{\alpha}\left(t_{j}\right)\left(1-u\left(t_{j}\right)\right)\right)=y_{j}^{1}-z_{j}^{1}$,

$\frac{\underline{i}^{\alpha}\left(t_{j+1}\right)-\underline{i}^{\alpha}\left(t_{j}\right)}{h}-\left(\beta \bar{n}^{-\alpha}\left(t_{j}\right) \bar{v}^{-\alpha}\left(t_{j}\right)\left(1-u\left(t_{j}\right)\right)-b \underline{i}^{\alpha}\left(t_{j}\right)\right)=y_{j}^{2}-z_{j}^{2}$

$\frac{\underline{v}^{\alpha}\left(t_{j+1}\right)-\underline{v}^{\alpha}\left(t_{j}\right)}{h}-\left(k i^{-\alpha}\left(t_{j}\right)-s \underline{v}^{\alpha}\left(t_{j}\right)\right)=y_{j}^{3}-z_{j}^{3}$

$\frac{\underline{n}^{\alpha}\left(t_{j+1}\right)-\underline{n}^{\alpha}\left(t_{j}\right)}{h}-\left(r-a \underline{n}^{\alpha}\left(t_{j}\right)-\beta \underline{n}^{\alpha}\left(t_{j}\right) \underline{v}^{\alpha}\left(t_{j}\right)\left(1-u\left(t_{j}\right)\right)\right)=y_{j}^{4}-z_{j}^{4}$

$\frac{\bar{i}^{\alpha}\left(t_{j+1}\right)-\bar{i}^{\alpha}\left(t_{j}\right)}{h}-\left(\beta \underline{n}^{\alpha}\left(t_{j}\right) \underline{v}^{\alpha}\left(t_{j}\right)\left(1-u\left(t_{j}\right)\right)-b \bar{i}^{\alpha}\left(t_{j}\right)\right)=y_{j}^{5}-z_{j}^{5}$

$\frac{\bar{v}^{\alpha}\left(t_{j+1}\right)-\bar{v}^{\alpha}\left(t_{j}\right)}{h}-\left(k \underline{i}^{\alpha}\left(t_{j}\right)-s \bar{v}^{\alpha}\left(t_{j}\right)\right)=y_{j}^{6}-z_{j}^{6}$

$\bar{n}^{-\alpha}\left(t_{0}\right)=1000 \alpha+1150(1-\alpha)$

$\bar{i}^{\alpha}\left(t_{0}\right)=1000 \alpha+850(1-\alpha)$

$\bar{v}^{\alpha}\left(t_{0}\right)=5 \alpha+6(1-\alpha)$

$\underline{n}^{\alpha}\left(t_{0}\right)=5 \alpha+4(1-\alpha)$

$\underline{i}^{\alpha}\left(t_{0}\right)=7000 \alpha+7250(1-\alpha)$

$\underline{v}^{\alpha}\left(t_{0}\right)=7000 \alpha+6750(1-\alpha)$

$y_{j}^{i} \geq 0, \quad z_{j}^{i} \geq 0$

$j=0, \ldots, n-1, \quad i=1, \ldots, 6$

It is assumed that $\alpha=0.2$, then software LINGO (version 11) is used to solve (14). Figures 2 show the boundaries of the $n, i$, and $v$ respectively. 
M. Najariyan, M. H. Farahi, and M. Alavian / TJMCS Vol .2 No.4 (2011) 639-649

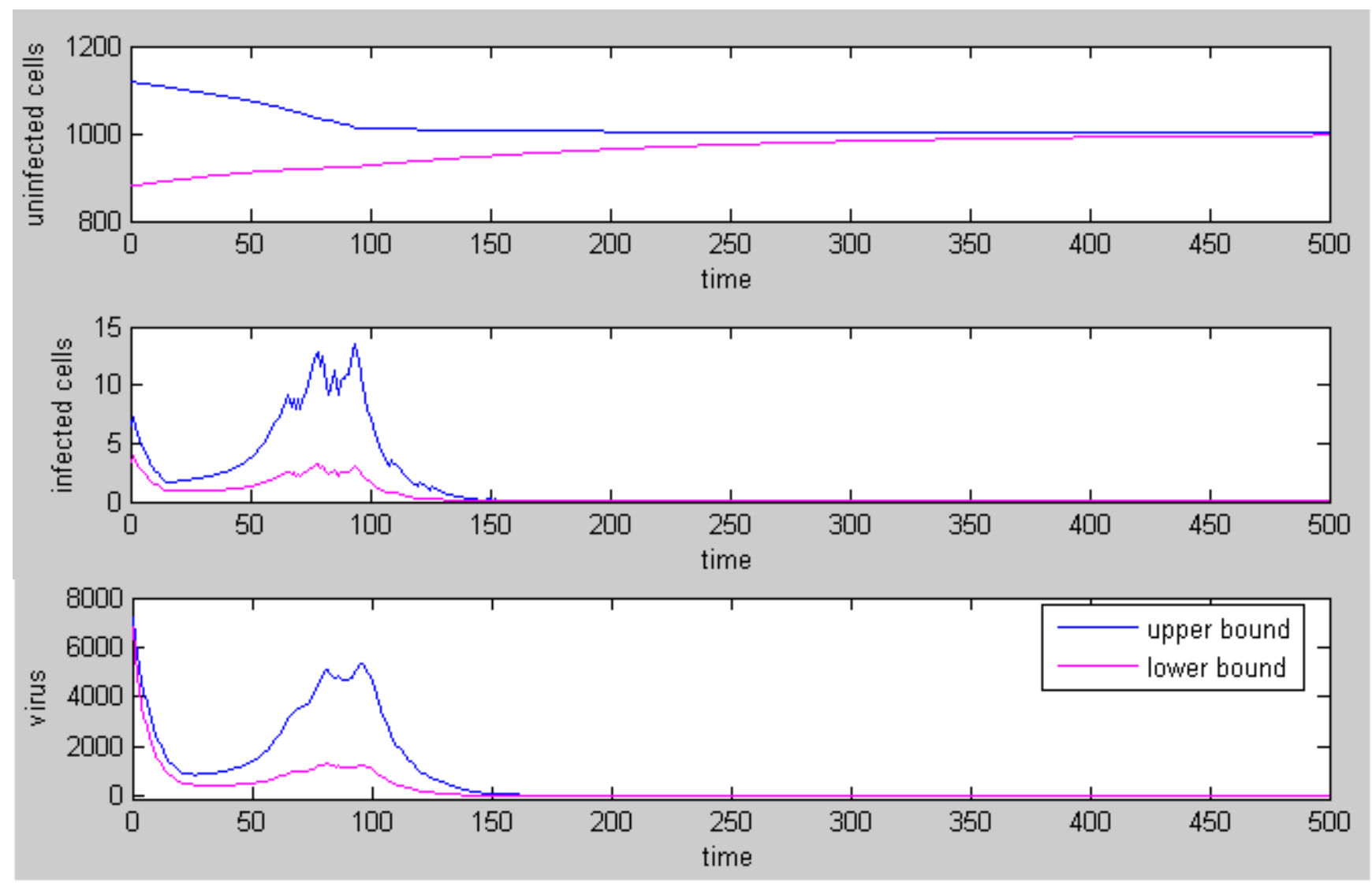

Figure 2. The solution for (14)

\section{Conclusion}

This paper has suggested a model of HIV infection with fuzzy differential equations. Since the proportion of the disease depend the sex, age, feeding,... one needs to consider the variables in equations as fuzzy variables. Also a fuzzy dynamical system is considered to control the HIV disease.

\section{References}

[1] Nowak, M. A., and May, R. M., "Mathematical biology of HIV infections, Antigenic variation and diversity threshold", Mathematical Biosciences, 106(1) pp. 1-21, 1991.

[2] Hone, A. N. W., and Painleve tests, "singularity structure and integrability", Arxiv preprint nlin, pp. 1-34, 2005.

[3] Capistran, M. A., and Solis, F. J.," On the modeling of long-term HIV-1 infection dynamics", Mathematical and computer modeling, 50 pp. 777-782, 2009.

[4] Nowak, M.A., and May, R. M., "Virus dynamic", Oxford University press, 2000.

[5] Pereslon, A. S., and Nelson, P. W., "Mathematical analysis of HIV-1 dynamics in vivo", SIAM, Rev.41, pp 3-44, 1999.

??[6] Pereslon, A. S., "Modeling the interaction of immune system with HIV", in: C. Castillo-Chavez (Ed.), Mathematical and statistical approaches to AIDS epidemiology, Springer, Berlin, 1989.

[7] Pereslon, A. S., Kirschner, D. E., and De Boer, R., "Dynamics of HIV infection of CD4 $4^{+}$T cells", Math, Biosci., 114, pp 81-125, 1993.

[8] Srivastava. P., and Chandra, P., "Modeling the dynamics of HIV and CD4 ${ }^{+}$T cells during primary infection", Nonlinear analysis: Real world applications, 11, pp. 612-618, 2010.

[9] Wein. L., Zenio, S., and Nowak, M.,"Dynamics multidrug therapies for HIV: A theoretic approach", Journal of Theoretical Biology, 35, pp. 15-29, 1997.

[10] Kirchner. D., and Lenhart, S., Serbin, S., "Optimal control of the chemotherapy of HIV", Journal of Mathematical Biology, 35, pp. 775-792, 1997. 


\section{Najariyan, M. H. Farahi, and M. Alavian / TJMCS Vol .2 No.4 (2011) 639-649}

[11] Mhawej. M., Moog, C. H., Biafore, F., and Francois C., "Control of HIV infection and drug dosage", Biomedical Signal Processing and control, 5, pp.45-52, 2010.

[12] Jafelice, R., Barros, L. C., Bassanezi, R. C., and gomide, F., "Fuzzy rules in asymptomatic HIV virus infected individuals model", Frontiers in Artifical Intelligence and Applications, Vol. 85, pp. 208-215, 2002a.

[13] Jafelice, R., Barros, L. C., Bassanezi, R. c. and Gomide, F., "Methodology to determine the evolution of asymptomatic HIV population using fuzzy set theory", (submitted for publication), 2003

[14] Barros, L., Bassanezi, R. C., and Leite, M. B., "The epidemiological models SI with fuzzy parameter of transmission", Comput. Math. Appl., 45, pp. 1619-1628, 2003.

[15] Ortega, N., Barros, L. C., and Massad, E.," Fuzzy gradual rules in epidemiology", Kyberentes: Int. J. Syst. Cybernetics, 32, pp. 460-477, 2003.

[16] Krivan, V., and Colombo, G.," A non-stochastic approach for modeling uncertainty in population dynamics". Bull. Math. Biol., 60, 1998.

[17] Diamond, P., and Kloeden, P., "Metric spaces of fuzzy sets", World scientific, Singapore, 1994.

[18] Puri, M., and Ralescu, D., "Differentials of fuzzy functions", J. Math. Anal. Appl., 91, pp.552-558, 1983.

[19] Bede, B., and Gal, S. G.," Generalizations of the differentiability of fuzzy-number-valued functions with applications to fuzzy differential equations", Fuzzy sets and systems, 151, pp. 581-599, 2005.

[20] Chalco-Cano, Y., and Roman-Flores, H., "One new solution of fuzzy differential equations", Chaos, Solitons \& Fractals, Vol. 38, no. 1, pp.112-119, 2008.

[21] Khastan, A., Bahrami, F., and Ivaz, K., "New results on multiple solutions for nth-order fuzzy differential equations under generalized differentiability", Boundary value problems, pp.1-13, 2009.

[22] Chalco-Cano, Y., Román-Flores, H., "On new solutions of fuzzy differential equations", Chaos, Solitons \& Fractals 38 pp.112-119, 2008.

[23] Nieto, J.J., Khastan, A., and Ivaz.K., "Numerical solution of fuzzy differential equations under generalized differentiability", Nonlinear Analysis: Hybrid Systems 3, pp.700-707, 2009

[24] Badakhshan, K. P., Kamyad, A. V., and Azemi, A.," Using AVK method to solve nonlinear problems with uncertain parameters", $A M C$, Vol. 189 ,pp. 27-34, 2007. 